Pacific Journal of Mathematic 


\title{
ORDERS OF FINITE ALGEBRAIC GROUPS
}

\author{
K. F. LAI
}

Let $\bar{G}$ be a simply connected simple algebraic group over a finite field $F_{q}$ of $q$ elements. The order of the group $\bar{G}\left(\boldsymbol{F}_{q}\right)$ of $\boldsymbol{F}_{q}$-rational points of $\bar{G}$ is well-known (cf: Steinberg, Carter). The proof makes use of the Bruhat decomposition and the study of polynomials invariant under the action the Weyl group. In this paper we deduce the order of $\bar{G}\left(F_{q}\right)$ from an explicit formula for the integral $M(s, \Lambda)$ which occurs in Langlands' theory of Eisenstein series.

First of all, according to a theorem of Lang $\bar{G}$ is quasi-split (cf: Lang [9], Satake [13] p. 105) and from Steinberg's theorem (cf: Steinberg [14], Kneser [6] p. 255) $\bar{G}$ is either a Chevalley group or a twisted group of one of the following types: ${ }^{2} A_{\mathfrak{l}}(\mathfrak{l} \geqq 2),{ }^{2} D_{\mathfrak{l}}(\mathfrak{l} \geqq 4)$, ${ }^{2} E_{6},{ }^{3} D_{4},{ }^{2} B_{2},{ }^{2} G_{2}$ and ${ }^{2} F_{4}$. To simplify matters we shall assume that the characteristic of $\boldsymbol{F}_{q}$ is not 2 and 3 and exclude groups of the type ${ }^{2} B_{2},{ }^{2} G_{2}$ and ${ }^{2} F_{4}$. Furthermore we can assume that there exists a quasi-split simple algebraic group $G$ defined over a $p$-adic number field $F$ such that the residue field of $F$ is isomorphic to $\boldsymbol{F}_{q}, G$ splits over an unramified Galois extension $E$ of $F$ and $G$ reduces modulo $p$ to $\bar{G}$ (cf: Weil [17]).

1. Fix a Haar measure $d x$ on $F$ such that the volume of the ring $R$ of $p$-adic integers in $F$ is one. Let $\omega$ be a left invariant highest $F$-differential form on $G$. Then $\omega$ and $d x$ determines a Haar measure on $G(F)$ which will also be denoted by $\omega$ (cf: Weil [17]).

LemMA 1. Let $m$ be the dimension of $\bar{G}$ and $\left|\bar{G}\left(\boldsymbol{F}_{q}\right)\right|$ be the order of $\bar{G}\left(\boldsymbol{F}_{q}\right)$. Then

$$
\left|\bar{G}\left(\boldsymbol{F}_{q}\right)\right|=q^{m} \int_{G(R)} \omega .
$$

This is proved in Weil [17] p. 22.

2. Let $B$ be a Borel subgroup of $G$ defined over $F$ and $A$ a maximal torus of $G$ in $B$. Then by assumption the Galois group Gal $(E / F)$ acts on the group $X(A)$ of rational characters of $A$. This gives rise to a representation

$$
\pi: \operatorname{Gal}(E / F) \longrightarrow \operatorname{Eng}(X(A) \underset{Z}{\otimes} \boldsymbol{Q}) .
$$


For $s \in \boldsymbol{C}$, let

$$
L(s, A)=\left(\operatorname{det}\left(I-q^{-s} \pi(\sigma)\right)\right)^{-1},
$$

where $\sigma$ is the Frobenius automorphism in $\operatorname{Gal}(E / F)$.

Let $N$ (resp. $\bar{N}$ ) be the unipotent radical of $B$ (resp. the Borel subgroup opposite to $B$ ). For $g \in G(F)$, if $g=n a k$ with $n \in N(F)$, $a \in A(F)$ and $k \in G(R)$, is the Iwasawa decomposition of $g$, then we denote $a$ by $a(g)$.

The data $(G, B, A)$ determined a root system $\Sigma$, a subset $\Sigma^{+}$of positive roots, and a basis $\Delta$ of $\Sigma$. Let $\rho$ be the half sum of the positive roots in $\Sigma$. Then $\rho$ defines a homomorphism on $A(F)$. (We denote this homomorphism also by $\rho$.) Let

$$
M=\int_{\bar{N}} \rho^{2}(a(\bar{n})) d \bar{n}
$$

$M$ is in fact a special value of the linear transformation $M(s, \Lambda)$ (cf: Langlands [10] p. 237) in the case $A$ is $\rho$ and $s$ is the Weyl group element which takes all the positive roots to negative roots. Rapoport ([12] p. 4-10) showed that

$$
M=L(1, A) \int_{G(R)} \omega .
$$

Comparing with (1) we get

$$
\left|\bar{G}\left(\boldsymbol{F}_{q}\right)\right|=q^{m} M L(1, A)^{-1} .
$$

We shall use this formula to calculate $\left|\bar{G}\left(\boldsymbol{F}_{q}\right)\right|$.

3. As we have already pointed out Steinberg's theorem implies that $G$ is obtained by twisting a $F$-split group $\widetilde{G}$ by a onecocycle

$$
\sigma \longrightarrow \dot{\phi}_{\sigma} \in Z^{1}(\operatorname{Gal}(E / F) \text {, Aut } \widetilde{G}) .
$$

And $\dot{\phi}_{\sigma}$ comes from the action of the Frobenius $\sigma$ on the Dynkin diagram of $G$. In fact, if we denote the action of $\sigma$ on $\alpha \in \Delta$ by $\sigma \alpha$, then this means that $\alpha \circ \dot{\phi}_{\sigma}=\sigma \alpha$. Moreover, since $\Delta$ forms a basis of $X(A) \otimes_{z} Q$, the representation $\pi$ in $\S 2$ is determined by the effect of $\pi(\sigma)$ on $\Delta$. And for $\alpha \in \Delta$ we have

$$
\pi(\sigma) \alpha=\sigma \alpha .
$$

Lemma 2. Let $\Omega_{0}$ be the set of orbits of $\sigma$ in $\Delta$. For $\rho \in \Omega_{0}$, $|\mathcal{O}|$ denotes the order of the orbit $\mathcal{O}$. Then for $s \in C$, 


$$
L(s, A)=\prod_{0 \in \Omega_{0}}\left(1-q^{-s|o|}\right)^{-1} .
$$

Proof. Suppose $\mathscr{O} \in \Omega_{0}$ and $|\mathscr{O}|=n$, then we can write

$$
\mathcal{O}=\left\{\alpha, \sigma \alpha, \cdots, \sigma^{n-1} \alpha\right\} \text {, for some } \alpha \in \Delta \text {. }
$$

Let $\pi(\sigma, \mathcal{O})$ be the restriction of $\pi(\sigma)$ to the subspace of $X(A) \otimes \boldsymbol{Q}$ spanned by $O$. Then with the basis in the order listed in (8), it is a trivial consequence of (6) that

$$
\begin{gathered}
\operatorname{det}\left(1-q^{-s} \pi(\sigma, \mathscr{O})\right) \\
=1-q^{-s n} .
\end{gathered}
$$

Finally noting that the action of $\sigma$ is broken into orbits, we see that the matrix of $\pi(\sigma)$ is broken into blocks on the diagonal. Since each block has determinant similar to that given in (9), the lemma is proved.

4. In $X(A) \otimes \boldsymbol{R}$ we choose a scalar product $(\cdot, \cdot)$ invariant under the Weyl group of the root system $\Sigma$. For every root $\alpha \in \Sigma$, we denote by $\hat{\alpha}: X(A) \otimes \boldsymbol{R} \rightarrow \boldsymbol{R}$ the linear form defined by

$$
\hat{\alpha}(\xi)=2(\xi, \alpha) /(\alpha, \alpha) \text {. }
$$

Let $\hat{\Sigma}=\{\hat{\alpha} \mid \alpha \in \Sigma\}$ and $\hat{\jmath}=\{\hat{\alpha} \mid \alpha \in \Delta\}$. Then $\hat{\Sigma}$ is a root system with base $\hat{\Delta}$. It is well-known that there exist a complex semisimple Lie algebra $\hat{g}$ and a Cartan subalgebra $\hat{a}$ of $\hat{g}$ such that the root system of $(\hat{\mathfrak{g}}, \hat{\mathfrak{a}})$ is $\hat{\Sigma}$ and the Cartan matrix of $\hat{g}$ is the transpose of

$$
\left(2\left(\alpha_{i}, \alpha_{j}\right) /\left(\alpha_{j}, \alpha_{j}\right)\right)_{i \leqq i, j \leq l}
$$

if $\lrcorner=\left\{\alpha_{1}, \cdots, \alpha_{l}\right\}$.

Choose $H_{1}, \cdots H_{l} \in \hat{a}$ so that $\xi\left(H_{i}\right)=\xi\left(\alpha_{i}\right)$ for all $\xi \in \operatorname{Hom}_{Z}(X(A)$, $Z)$. In $\hat{\mathrm{g}}$ choose root vectors $X_{ \pm} \hat{\alpha}_{i}$ belonging to $\pm \hat{\alpha}_{i}$ such that

$$
\left[X_{\hat{\alpha}_{i}}, X_{-\hat{\alpha}_{i}}\right]=H_{\imath} 1 \leqq i \leqq \ell .
$$

If $\sigma \in \operatorname{Gal}(E / F)$, let $\sigma \widehat{\alpha}=\widehat{\sigma \alpha}$ for $\alpha \in \Delta$. Extend this action linearly to $\hat{\Sigma}$. This means that the orbits of $\sigma$ in $\hat{\Sigma}$ and $\Sigma$ corresponds bijectively. Moreover there is an automorphism (also denoted by) $\sigma$ of the Lie algebra $\hat{g}$ so that

$$
\sigma\left(H_{i}\right)=H_{j} \text { and } \sigma X_{\hat{\alpha}_{i}}=X_{\hat{\alpha}_{j}},
$$

if $\sigma \widehat{\alpha}_{i}=\widehat{\alpha}_{j}$ (cf: Jacobson [5] Chap. VII).

Let $\hat{\Omega}$ (resp. $\hat{\Omega}_{0}$ ) denote the set of orbits of the Frobenius automorphism $\sigma\left(\varepsilon \operatorname{Gal}(E / F)\right.$ ) acting on $\hat{\Sigma}^{+}$(resp. $\left.\hat{\Delta}\right)$. Let $\hat{\Omega}_{1}$ be the set of those elements of $\hat{\Omega}$ not in $\hat{\Omega}_{0}$. We shall pick a representa- 
tive in each orbit and label each orbit by the corresponding representative. Suppose

$$
\mathcal{O}(\widehat{\alpha})=\left\{\hat{\alpha}, \sigma \widehat{\alpha}, \cdots, \sigma^{n-1} \widehat{\alpha}\right\}
$$

is such an orbit, we use $n(\widehat{\alpha})$ to denote the order of the orbit $\mathscr{O}(\hat{\alpha})$ and define the number $\eta(\hat{\alpha})$ by

$$
\sigma^{n} X_{\hat{\alpha}}=\eta(\widehat{\alpha}) X_{\hat{\alpha}} .
$$

5. Following Bhanu-Murthy [1], Gindikin-Karpelevich [4] and Langlands [11], Lai [8] (p. 56) has calculated the integral (3)-in the notations of previous section-

$$
M=\prod_{0, \hat{\alpha}) \in \hat{Q}} \frac{1-\eta(\hat{\alpha}) q^{-n(\hat{\alpha})(1+\hat{\alpha}(\rho))}}{1-\eta(\hat{\alpha}) q^{-n}(\hat{\alpha}) \hat{\alpha}(\rho)} .
$$

THEOREM. Let $\bar{G}$ be a simply connected simple algebraic group over a finite field $\boldsymbol{F}_{q}$ of $q$ elements. $\bar{G}$ not of the types ${ }^{2} B_{2},{ }^{2} G_{2}$ and ${ }^{2} F_{4}$. Then the order of the $\boldsymbol{F}_{q}$-rational points is given by

$$
\left|\bar{G}\left(\boldsymbol{F}_{q}\right)\right|=q^{m} \frac{\prod_{\rho \hat{\alpha}) \in \hat{\hat{\alpha}}}\left(1-\eta(\hat{\alpha}) q^{-n(\hat{a})(1+\hat{\alpha}(\rho))}\right)}{\prod_{\sigma(\hat{\alpha}) \in \hat{\hat{L}_{1}}}\left(1-\eta(\hat{\alpha}) q^{-n \hat{\alpha} \hat{\alpha}) \hat{\alpha}(\rho)}\right)} .
$$

More specifically, if $\bar{G}$ is a Chevalley group and $d_{i}, 1 \leqq i \leqq \ell$ its exponents then

$$
\left|\bar{G}\left(\boldsymbol{F}_{q}\right)\right|=q^{m} \prod_{i=1}^{l}\left(1-q^{-d_{i}}\right) .
$$

For the twisted groups we have

$$
\begin{gathered}
\left|{ }^{2} A_{\mathfrak{r}}\left(\boldsymbol{F}_{q}\right)\right|=q^{l(l+1) / 2} \prod_{i=2}^{l+1}\left(q^{i}-(-1)^{i}\right) \\
\left|{ }^{2} D_{l}\left(\boldsymbol{F}_{q}\right)\right|=q^{l(l-1)}\left(q^{l}+1\right) \prod_{i=1}^{l-1}\left(q^{2 i}-1\right) \\
\left|{ }^{3} D_{4}\left(\boldsymbol{F}_{q}\right)\right|=q^{12}\left(q^{2}-1\right)\left(q^{6}-1\right)\left(q^{8}+q^{4}+1\right) \\
\left|{ }^{2} E_{6}\left(\boldsymbol{F}_{q}\right)\right|=q^{36}\left(q^{2}-1\right)\left(q^{5}+1\right)\left(q^{6}-1\right)\left(q^{8}-1\right)\left(q^{9}+1\right)\left(q^{12}-1\right) .
\end{gathered}
$$

Proof. As we have already pointed out the orbits of $\sigma$ in $\Sigma$ and $\hat{\Sigma}$ corresponds, so we can substitute (7) and (11) into (5) to get (12) if we note that $\hat{\alpha}(\rho)=1$ and $\eta(\hat{\alpha})=1$ for $\hat{\alpha} \in \hat{\Delta}$. We shall use (12) to calculate $\left|\bar{G}\left(\boldsymbol{F}_{q}\right)\right|$ case by case.

6. In case $G$ is a Chevalley group the calculation is well-known. 
We shall only sketch the arguments. The exponents $d_{i}$ are integers so that $\prod_{i=1}^{l}\left(1+t^{2 d_{i-1}}\right)$ is the Poincare polynomial of the compact real form of $G$. According to an observation of Shapiro and of Steinberg [15] (for proof see Kostant [7]), the set of numbers

$$
\{1+\hat{\alpha}(\rho)\}_{\hat{\alpha} \in \hat{2}^{+}}
$$

is the same as the set of numbers

$$
\{\widehat{\alpha}(\rho)\}_{\hat{\alpha} \in \hat{\Sigma}+-\hat{u}} \cup\left\{d_{i}\right\}_{1 \leq i \leq l} .
$$

Together with the fact that $\sigma$ acts trivially so that $\hat{\Omega}=\hat{\Sigma}^{+}, \hat{\Omega}_{0}=\hat{\Delta}$ and $n(\hat{\alpha})=\eta(\hat{\alpha})=1$, we get from (12) immediately the formula (13). This formula is due to Chevalley [3].

7. The case of the twisted group ${ }^{2} A_{2 k}$ i.e., the group obtained from a group of type $A_{2 k}$ by a twisting by the Frobenius of the quadratic extension $E / F$. The Dynkin diagram is

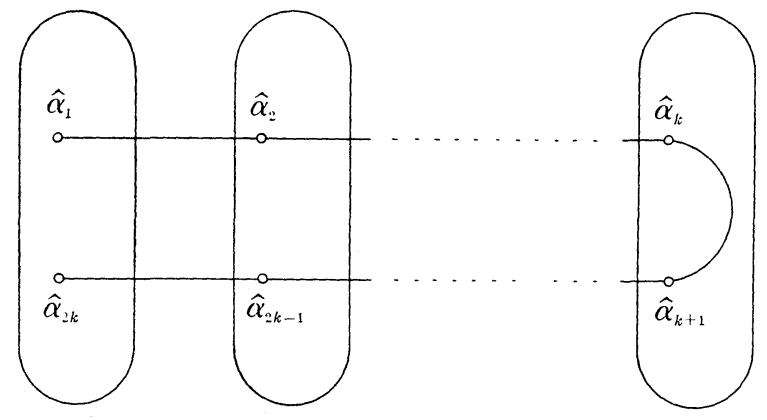

We have circled together the elements of the same orbit.

Hereafter we shall use an abbreviation for a root by indicating only the coefficients. For example in the case of ${ }^{2} A_{4}$, the symbol

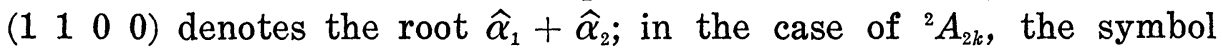
$(0 \cdots 011: 110 \cdots 0)$ denotes the root $\hat{\alpha}_{k-1}+\hat{\alpha}_{k}+\hat{\alpha}_{k+1}+\hat{\alpha}_{k+2}$.

By looking at root table it is easy to see that the orbits in $\hat{\Sigma}^{+}$ have either one or two roots. In fact the one element orbits: $\mathcal{O}(\hat{\alpha})$ are $(0 \cdots 01 \vdots 10 \cdots 0),(0 \cdots 011 \vdots 110 \cdots 0), \cdots(01 \cdots 1 \vdots 1 \cdots 10)$ and $(1 \cdots 11 \cdots 1)$; the respective values of $\hat{\alpha}(\rho)$ are $2,4, \cdots, 2 k-1$ and $2 k$. The value of $\eta(\hat{\alpha})$ in each of these cases is -1 . The rest of the orbits have two roots in each of them and in all these cases $\eta(\hat{\alpha})=1$. It is easy to see that $\widehat{\alpha}(\rho)$ equal to the number of 1 's in the symbol of $\hat{\alpha}$. One now writes down all the factors occurring in the right hand side of (12). After removing those terms that occur both in the numerator and denominator we get

$$
\frac{q^{m}\left(1-q^{-4}\right)\left(1+q^{-3}\right) \cdots\left(1-q^{-4 k}\right)\left(1+q^{-(2 k+1)}\right)}{\left(1+q^{-2}\right) \cdots\left(1+q^{-2 k}\right)}=q^{m} \prod_{i=2}^{2 k+1}\left(1-(-1)^{i} q^{-i}\right) .
$$


Since $m=4\left(k^{2}+k\right)$, we get (14) for the case $\mathfrak{l}=2 k$.

8. Similar computations can be made in the case $G$ is of the type ${ }^{2} A_{2 k-1}$. The orbits of the twisted action on the Dynkin diagram are
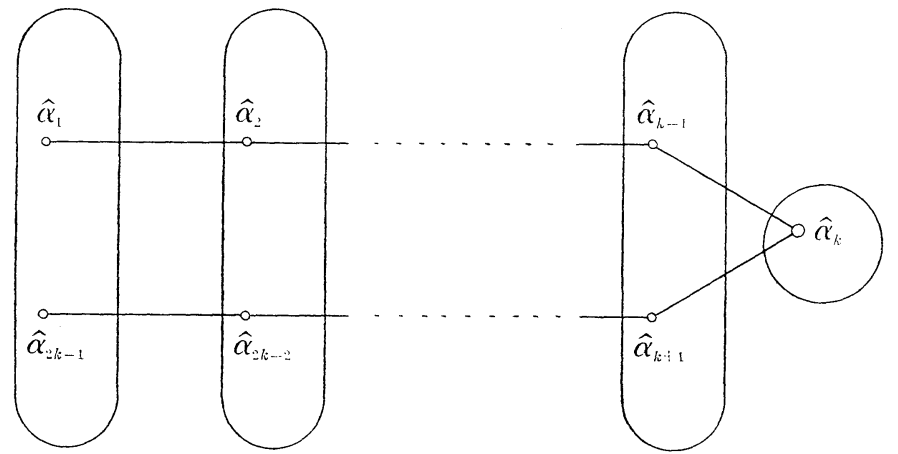

The right hand side of (12) after removing like terms from the numerator and denominator is

$$
\begin{aligned}
\frac{q^{m}\left(1-q^{-2}\right)\left(1-q^{-6}\right) \cdots\left(1-q^{-2(2 k-1)}\right)\left(1-q^{-2 k}\right)}{\left(1-q^{-3}\right) \cdots\left(1-q^{-(2 k-1)}\right)} & =q^{m} \prod_{i=2}^{2 k}\left(1-(-1)^{i} q^{-i}\right) \\
& =q^{k(2 k-1)} \prod_{i=2}^{2 k}\left(q^{i}-(-1)^{i}\right) .
\end{aligned}
$$

This is just (14) when $l=2 k-1$.

9. As for the case of ${ }^{2} D_{\mathrm{r}}$, the Dynkin diagram is

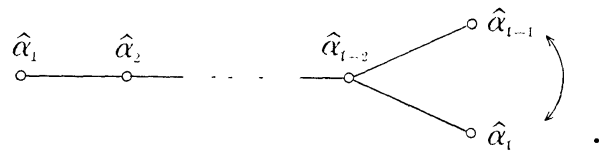

We have used an arrow to indicate the action of $\sigma$. That is $\sigma$ interchanges $\hat{\alpha}_{1-1}$ and $\hat{\alpha}_{\mathfrak{r}}$ and fixes the rest of the simple roots. Using root tables to calculate the right hand side of (12), we found, after removing the like terms from the numerator and denominator, that the denominator has only one term $1-q^{-1}$ corresponding to the root $\hat{\alpha}_{1}+\cdots+\widehat{\alpha}_{i}$; whereas, the numerator contains the terms $1-q^{-2}, 1-q^{-4}, 1-q^{-6}, 1-q^{-8}, \cdots, 1-q^{-(21-2)}$ corresponding to the roots $\hat{\alpha}_{1}, \hat{\alpha}_{1}+\hat{\alpha}_{2}+\hat{\alpha}_{3}, \hat{\alpha}_{1-3}+2 \widehat{\alpha}_{1-2}+\widehat{\alpha}_{1-1}+\hat{\alpha}_{1}, \hat{\alpha}_{1-4}+2\left(\hat{\alpha}_{1-3}+\right.$ $\left.\hat{\alpha}_{1-2}\right)+\hat{\alpha}_{1-1}+\hat{\alpha}_{1}, \cdots, \hat{\alpha}_{1}+2\left(\hat{\alpha}_{2}+\cdots+\hat{\alpha}_{1-2}\right)+\hat{\alpha}_{\mathfrak{r}-1}+\hat{\alpha}_{\mathfrak{r}}$ respectively, and the term $1-q^{-2 t}$ corresponding to the orbit $\left\{\left(\hat{\alpha}_{1}+\cdots+\hat{\alpha}_{1-1}\right.\right.$, $\left.\hat{\alpha}_{\mathfrak{t}}+\cdots+\hat{\alpha}_{\mathrm{t}-2}+\hat{\alpha}_{\mathfrak{t}}\right)$. As a result we get

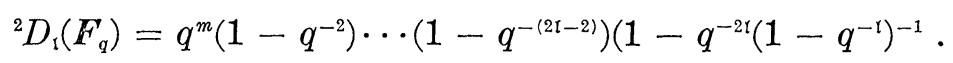


Since $m=2 \mathfrak{l}^{2}-\mathfrak{l}$ we get (15) immediately.

10. The next case is ${ }^{3} D_{4}$. The Dynkin diagram with the action of Frobenius indicated by arrows is:

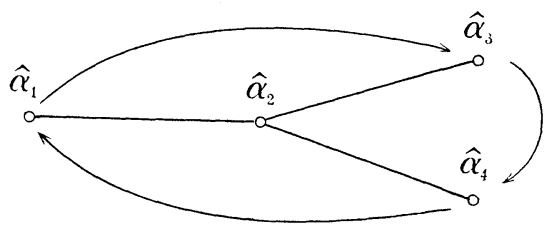

Direct calculation shows that for all orbits $\eta(\hat{\alpha})=1$. The values of $n(\hat{\alpha})$ and $\hat{\alpha}(\rho)$ are given in the following table.

\begin{tabular}{|c|c|c|c|c|c|c|}
\hline & $\mathcal{O}(\hat{\alpha})_{1}$ & $\sigma\left(\hat{\alpha}_{2}\right)$ & $\mathcal{O}\left(\hat{\alpha}_{1}+\hat{\alpha}_{2}\right)$ & $\mathcal{O}\left(\hat{\alpha}_{1}+\hat{\alpha}_{2}+\hat{\alpha}_{3}\right)$ & $\mathcal{O}\left(\hat{\alpha}_{1}+\hat{\alpha}_{2}+\hat{\alpha}_{3}+\hat{\alpha}_{4}\right)$ & $\mathscr{O}\left(\hat{\alpha}_{1}+2 \hat{\alpha}_{2}+\hat{\alpha}_{3}+\hat{\alpha}_{4}\right)$ \\
\hline$n(\hat{\alpha})$ & 3 & 1 & 3 & 3 & 1 & 1 \\
\hline$\widehat{\alpha}(\rho)$ & 1 & 1 & 2 & 3 & 4 & 5 \\
\hline
\end{tabular}

In this case $m=28$. The right hand side of (12) is

$$
\begin{gathered}
\frac{q^{28}\left(1-q^{-6}\right)\left(1-q^{-2}\right)\left(1-q^{-9}\right)\left(1-q^{-12}\right)\left(1-q^{-5}\right)\left(1-q^{-6}\right)}{\left(1-q^{-6}\right)\left(1-q^{-9}\right)\left(1-q^{-4}\right)\left(1-q^{-5}\right)} \\
=q^{12}\left(q^{2}-1\right)\left(q^{6}-1\right)\left(q^{8}+q^{4}+1\right)
\end{gathered}
$$

and we get (16).

11. The last case is ${ }^{2} E_{6}$. The Dynkin diagram with Galois action indicated by arrows is

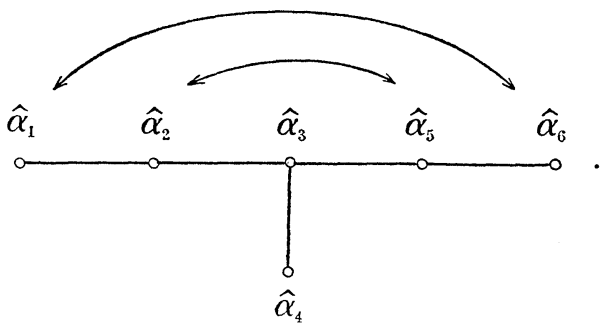

$\hat{\Sigma}^{+}$has 36 elements breaking up into 24 orbits with atmost 2 elements in each orbit; $\eta(\hat{\alpha})$ is always 1 . Let us write $\left(k_{1} \cdots k_{6}\right)$ for the root

$$
\hat{\alpha}=k_{1} \hat{\alpha}_{1}+\cdots+k_{6} \hat{\alpha}_{6} .
$$

Then $\hat{\alpha}(\rho)=k_{1}+\cdots+k_{6}$. The 2 elements orbit are: 


$\begin{array}{ll}\{(100000),(000001)\}, & \{(010000),(000010)\}, \\ \{(110000),(000011)\}, & \{(111000),(001011)\}, \\ \{(111100),(001111)\}, & \{(111110),(011111)\}, \\ \{(011000),(001010)\}, & \{(011100),(001110)\}, \\ \{(011011),(111010)\}, & \{(122110),(012121)\}, \\ \{(112110),(012111)\}, & \{(112121),(122111)\} .\end{array}$

The rest of the orbits are

$$
\begin{aligned}
& \text { (001000), (000100), (111111), (111011), } \\
& \text { (011010), (001100), (012110), (011110), } \\
& \text { (123221), (123121), (122121), (112111). }
\end{aligned}
$$

Evaluating the right hand side of (12) leads to

$$
q^{78}\left(1-q^{-2}\right)\left(1+q^{-5}\right)\left(1-q^{-6}\right)\left(1-q^{-8}\right)\left(1+q^{-9}\right)\left(1-q^{-12}\right)
$$

which is just (17). This completes the proof of the theorem.

12. It is sometimes convenient to express formula (12) in terms of determinants. Let $h$ be an element in the adjoint group of $\hat{g}$ such that

$$
h \cdot X_{\hat{\alpha}}=q^{-1} X_{\hat{\alpha}}
$$

for $\hat{\alpha} \in \hat{\Delta}$. Take an orbit $\mathcal{O}(\hat{\alpha})$ of the action of the Frobenius automorphism $\sigma$. As $\sigma$ is an isometry on the real vector space spanned by $\hat{\Sigma}$ (cf: Carter [2] p. 201), we have

$$
h \cdot X_{\hat{\beta}}=q^{-\hat{\alpha}(\rho)} X_{\hat{\beta}}
$$

for $\widehat{\beta} \in \mathcal{O}(\widehat{\alpha})$.

Let $\hat{\mathfrak{n}}(\hat{\alpha})$, $\hat{\mathfrak{H}}$ be the subalgebras of $\hat{\mathfrak{g}}$ spanned by $\left\{X_{\hat{\beta}} \mid \widehat{\beta} \in \mathcal{O}(\hat{\alpha})\right\}$, $\left\{X_{\hat{\beta}} \mid \widehat{\beta} \in \hat{\Sigma}^{+}\right\}$respectively. Then $\sigma$ on $\hat{\mathfrak{n}}(\hat{\alpha})$ has matrix

$$
\left(\begin{array}{c|c}
0 \cdots & \eta(\widehat{\alpha}) \\
\hline I & \vdots \\
& 0
\end{array}\right)
$$

where $I$ is the identity matrix. The matrix of $\sigma$ on $\hat{\mathfrak{t}}$ is broken into such blocks on the diagonal and the blocks are parametrized by $\hat{\Omega}$. Thus we get 


$$
\begin{gathered}
\prod_{\langle\hat{\alpha}) \in \hat{\Omega}}\left(1-\eta(\hat{\alpha}) q^{-m(\hat{\alpha})(1+\hat{\alpha}(\rho))}\right) \\
=\operatorname{det}_{\hat{\mathfrak{n}}}\left(1-q^{-1} \sigma h\right)
\end{gathered}
$$

where $\operatorname{det}_{\hat{n}}$ means the determinant of $1-q^{-1} \sigma h$ acting on $\hat{\mathfrak{n}}$.

Let $\hat{\mathfrak{r}}_{1}$ be the subalgebra of $\hat{\mathrm{g}}$ spanned by $X_{\hat{\beta}}$ for $\hat{\beta} \in \mathcal{O}(\hat{\alpha})$ and $\mathcal{O}(\hat{\alpha}) \in \hat{\Omega}_{1}$. Then similar argument leads to

$$
\begin{array}{r}
\prod_{\sigma(\hat{\alpha}) \in \hat{\delta_{1}}}\left(1-\eta(\hat{\alpha}) q^{-n(\hat{\alpha}) \hat{\alpha}(\rho)}\right) \\
=\operatorname{det}_{{\hat{n_{1}}}_{1}}(1-\sigma h) .
\end{array}
$$

The following corollary is now immediate.

CoROLLARY.

$$
\left|\bar{G}\left(\boldsymbol{F}_{q}\right)\right|=\frac{q^{m} \operatorname{det}_{\hat{\mathfrak{n}}}\left(1-q^{-1} \sigma h\right)}{\operatorname{det}_{\hat{\mathrm{n}}_{1}}(1-\sigma h)}
$$

13. Finally we would like to point out that our formula agrees with the standard formula for the order of finite twisted groups (as given for example in Carter [2]).

For $1 \leqq i \leqq \mathfrak{l}$, we can assume that $\left\{H_{i}, X_{\hat{\alpha}_{i}}, X_{-\hat{\alpha}_{i}}\right\}$ forms a basis of a 3-dimensional simple Lie algebra. Put

$$
H=\sum_{i} H_{i}, X=\sum_{i} X_{\hat{\alpha}_{i}}, Y=\sum_{i} X_{-\hat{\alpha}_{i}} .
$$

and let $\hat{\mathfrak{s}}$ be the 3 -dimensional subalgebra with basis $\{H, X, Y\}$. If $d_{1}, \cdots, d_{1}$ are the exponents of $G$, then according to Kostant [7], $\hat{\mathfrak{g}}$ as an $\hat{\mathfrak{s}}$-module under the adjoint representation decomposes into irreducible submodules $\hat{\mathfrak{D}}_{i}$ of dimensions $2 d_{i}-1$ for $1 \leqq i \leqq \mathfrak{l}$. On the other hand it is clear from paragraph 4 that $\sigma$ leaves invariant $H, X$ and $Y$ and hence we can arrange the decomposition $\hat{\mathfrak{g}}=\oplus \hat{\mathrm{\delta}}_{i}$ in such a way that $\sigma$ leaves each factor $\hat{\mathfrak{D}}_{i}$ invariant and acts on $\hat{\mathfrak{D}}_{i}$ by the root of unity $\varepsilon_{i}$. Moreover the element $h$ of paragraph 13 belongs to the connected subgroup of $A d(\hat{\mathfrak{g}})$ with Lie algebra $\hat{\mathfrak{s}}$ and so $h$ also leaves each submodule $\hat{\mathfrak{D}}_{i}$ invariant.

If $\hat{\alpha} \in \hat{\Sigma}^{+}$let $o(\alpha)$ be the sum of the coefficients of $\hat{\alpha}$ relative to the basis $\hat{\Delta}$. Then we group the eigenvalues $\left\{q^{-o(\alpha)} \mid \hat{\alpha} \in \hat{\Sigma}^{+}\right\}$of the restriction of $h$ to $\hat{\mathfrak{n}}$ according to the decomposition $\hat{\mathfrak{g}}=\oplus \hat{\mathfrak{d}}_{i}$. In fact, let $\Delta_{i}$ be the set of $\hat{\alpha} \in \hat{\Sigma}^{+}$such that the eigenvector corresponding to the eigenvalue $q^{-o(\hat{\alpha})}$ lies in $\hat{\mathfrak{b}}_{i}$. The corresponding set with $\hat{\Sigma}^{+} \backslash \Delta^{+}$replacing $\hat{\Sigma}^{+}$will be denoted by $\Delta_{i}^{\prime}$. Then we get immediately, 


$$
\operatorname{det}_{\hat{n}}\left(1-q^{-1} \sigma h\right)=\prod_{i=1}^{l} \prod_{\hat{\alpha} \in \Delta_{i}}\left(1-\varepsilon_{i} q^{-\langle o(\hat{\alpha})+1\rangle}\right)
$$

and

$$
\operatorname{det}_{\mathfrak{n}_{1}}(1-\sigma h)=\prod_{i=1}^{l} \prod_{\hat{\alpha} \in \Delta_{i}^{\prime}}\left(1-\varepsilon_{i} q^{-o(\alpha)}\right) .
$$

The procedure of Shapiro as proved in Kostant [7] says that for $1 \leqq i \leqq \mathfrak{l}$, the set of integers

$$
\{1+o(\widehat{\alpha})\}_{\hat{\alpha} \in \Lambda_{i}}
$$

is the same as the set of integers

$$
\{o(\hat{\alpha})\}_{\hat{\alpha} \in \Delta_{i}^{\prime}} \cup\left\{d_{i}\right\}
$$

It is immediate from these formulas that (19) yields

$$
\left|\bar{G}\left(\boldsymbol{F}_{q}\right)\right|=q^{m} \prod_{i=1}^{l}\left(1-\varepsilon_{i} q^{-d_{i}}\right)
$$

This is the standard formula for the order of finite twisted groups.

14. We would like to thank the referee for his suggestions in particular with regard to paragraphs 12 and 13.

\section{REFERENCES}

1. T. S. Bhanu-Murthy, Plancherel's measure for the factor space $S L(n, R) / S O(n, R)$, Soviet Math. Dokl., 1 (1960), 860.

2. R. W. Carter, Simple Groups of Lie Type, Wiley, New York, 1972.

3. C. Chevalley, Sur certains groupes simples, Tôhoku Math. J., 7 (1955), 14-66.

4. S. G. Gindikin and F. I. Karpelevic, Plancherel measure for Riemannian symmetric spaces, Soviet Math. Dokl., 3 (1962), 962-965.

5. N. Jacobson, Lie Algebras, New York, Interscience, 1962.

6. M. Kneser, Semisimple algebraic groups, Proc. Conf. Alg. Number Theory, London Math. Soc., (1967), 250-264.

7. B. Kostant, The principal three dimensional subgroup, Amer. J. Math., 81 (1959), 973-1032.

8. K. F. Lai, On the Tamagawa number of quasi-split groups, Yale Thesis, 1974.

9. S. Lang, Algebraic groups over finite fields, Amer. J. Math., 78 (1956), 555-563.

10. R. P. Langlands, Eisenstein series, AMS Proc. Sym. Pure Math., 9 (1966), 235-252.

11. - Euler Products, Yale University Press, New Haven, 1971.

12. M. Rapoport, Determination du nombre de Tamagawa des groupes semi-simples quasi-deployes sur des corps de nombres, preprint.

13. I. Satake, Classification Theory of Semi-Simple Algebraic Groups, Marcel Dekker, New York, 1971.

14. R. Steinberg, Variations on a theme of Chevalley, Pacific J. Math., 9 (1959), 875-891. 
15. R. Steinberg, Finite reflection groups, Trans. Amer. Math. Soc., 91 (1959), 493.

16. - Lectures on Chevalley groups, Yale University, New Haven, 1967.

17. A. Weil, Adeles and algebraic groups, IAS Princeton, 1961.

Received May 2, 1979.

Chinese University of HoNG Kong

Shatin, Hong Kong 



\section{PACIFIC JOURNAL OF MATHEMATICS}

EDITORS

DONALD BABBITT (Managing Editor)

University of California

Los Angeles, CA 90024

Hugo RossI

University of Utah

Salt Lake City, UT 84112

C. C. MOORE and ANDREW OGG

University of California

Berkeley, CA 94720

\section{J. DugundjI}

Department of Mathematics

University of Southern California

Los Angeles, CA 90007

R. FINN and J. MILGRAM

Stanford University

Stanford, CA 94305

\section{ASSOCIATE EDITORS}
R. ARENS
E. F. BECKENBACH
B. H. NEUManN
F. WOLF
K. YoSHIDA

\section{SUPPORTING INSTITUTIONS}

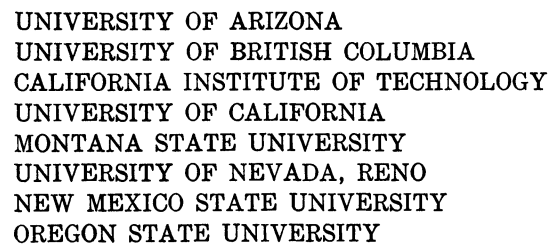

UNIVERSITY OF ARIZONA

UNIVERSITY OF BRITISH COLUMBIA

CALIFORNIA INSTITUTE OF TECHNOLOGY

UNIVERSITY OF CALIFORNIA

MONTANA STATE UNIVERSITY

UNIVERSITY OF NEVADA, RENO

NEW MEXICO STATE UNIVERSITY

OREGON STATE UNIVERSITY

\author{
UNIVERSITY OF OREGON \\ UNIVERSITY OF SOUTHERN CALIFORNIA \\ STANFORD UNIVERSITY \\ UNIVERSITY OF HAWAII \\ UNIVERSITY OF TOKYO \\ UNIVERSITY OF UTAH \\ WASHINGTON STATE UNIVERSITY \\ UNIVERSITY OF WASHINGTON
}

The Supporting Institutions listed above contribute to the cost of publication of this Journal, but they are not owners or publishers and have no responsibility for its content or policies.

Mathematical papers intended for publication in the Pacific Journal of Mathematics should be in typed form or offset-reproduced, (not dittoed), double spaced with large margins. Please do not use built up fractions in the text of the manuscript. However, you may use them in the displayed equations. Underline Greek letters in red, German in green, and script in blue. The first paragraph or two must be capable of being used separately as a synopsis of the entire paper. Please propose a heading for the odd numbered pages of less than 35 characters. Manuscripts, in triplicate, may be sent to any one of the editors. Please classify according to the scheme of Math. Reviews, Index to Vol. 39. Supply name and address of author to whom proofs should be sent. All other communications should be addressed to the managing editor, or Elaine Barth, University of California, Los Angeles, California, 90024.

50 reprints to each author are provided free for each article, only if page charges have been substantially paid. Additional copies may be obtained at cost in multiples of 50 .

The Pacific Journal of Mathematics is issued monthly as of January 1966. Regular subscription rate: $\$ 102.00$ a year (6 Vols., 12 issues). Special rate: $\$ 51.00$ a year to individual members of supporting institutions.

Subscriptions, orders for numbers issued in the last three calendar years, and changes of address shoud be sent to Pacific Journal of Mathematics, P.O. Box 969, Carmel Valley, CA 93924, U.S.A. Old back numbers obtainable from Kraus Periodicals Co., Route 100, Millwood, NY 10546.

\section{PUBLISHED BY PACIFIC JOURNAL OF MATHEMATICS, A NON-PROFIT CORPORATION}

Printed at Kokusai Bunken Insatsusha (International Academic Printing Co., Ltd.). 8-8, 3-chome, Takadanobaba, Shinjuku-ku, Tokyo 160, Japan. 


\section{Pacific Journal of Mathematics}

\section{Vol. 97, No. $2 \quad$ February, 1981}

Patrick Robert Ahern and N. V. Rao, A note on real orthogonal measures . . . . . 249

Kouhei Asano and Katsuyuki Yoshikawa, On polynomial invariants of fibered

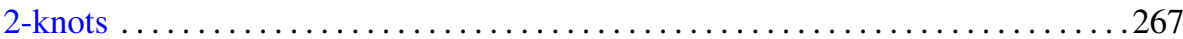

Charles A. Asmuth and Joe Repka, Tensor products for $S L_{2}(\mathscr{K})$. I.

Complementary series and the special representation

Gary Francis Birkenmeier, Baer rings and quasicontinuous rings have a

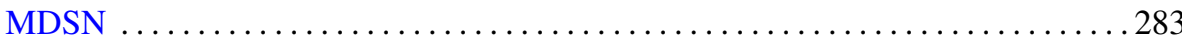

Hans-Heinrich Brungs and Günter Törner, Right chain rings and the generalized

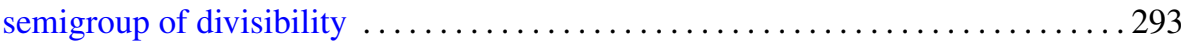

Jia-Arng Chao and Svante Janson, A note on $H^{1} q$-martingales . . . . . . . . . 307

Joseph Eugene Collison, An analogue of Kolmogorov's inequality for a class of

additive arithmetic functions

Frank Rimi DeMeyer, An action of the automorphism group of a commutative ring on its Brauer group

H. P. Dikshit and Anil Kumar, Determination of bounds similar to the Lebesgue

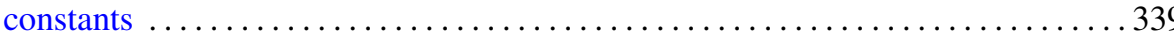

Eric Karel van Douwen, The number of subcontinua of the remainder of the plane

D. W. Dubois, Second note on Artin's solution of Hilbert's 17th problem. Order spaces

Daniel Evans Flath, A comparison of the automorphic representations of GL(3) and its twisted forms

Frederick Michael Goodman, Translation invariant closed $*$ derivations

Richard Grassl, Polynomials in denumerable indeterminates

K. F. Lai, Orders of finite algebraic groups

George Kempf, Torsion divisors on algebraic curves

Arun Kumar and D. P. Sahu, Absolute convergence fields of some triangular matrix methods

Elias Saab, On measurable projections in Banach spaces

Chao-Liang Shen, Automorphisms of dimension groups and the construction of AF algebras

Barry Simon, Pointwise domination of matrices and comparison of $\Phi_{p}$ norms

Chi-Lin Yen, A minimax inequality and its applications to variational inequalities

Stephen D. Cohen, Corrections to: "The Galois group of a polynomial with two indeterminate coefficients"

Phillip Schultz, Correction to: "The typeset and cotypeset of a rank 2 abelian group"

Pavel G. Todorov, Correction to: "New explicit formulas for the $n$th derivative of composite functions"

Douglas S. Bridges, Correction to: "On the isolation of zeroes of an analytic function" 